\title{
An improved experimental methodology to evaluate the effectiveness of protective gloves against nanoparticles in suspension
}

\author{
Ludwig Vinches $(\varangle)$, Mohamed Zemzem, Stéphane Hallé \\ Dept. of Mechanical Engineering, École de technologie supérieure \\ 1100 Notre-Dame Ouest, Montreal (QC) H3C 1 K3 Canada \\ Caroline Peyrot, Kevin J. Wilkinson \\ Dept. of Chemistry, University of Montreal \\ C.P. 6128, succ. Centre-ville, Montreal (QC) H3C 3J7 Canada \\ Nathalie Tufenkji \\ Dept. of Chemical Engineering, McGill University \\ 3610 University St., Montreal (QC) H3A OC5 Canada
}

$\bowtie$ ludwig.vinches@gmail.com 


\begin{abstract}
Recent studies underline the potential health risks associated to the "nano" revolution, particularly for the workers who handle engineered nanoparticles (ENPs) that can be found in the formulation of several commercial products. Although many Health \& Safety agencies recommend the use of protective gloves against chemicals, few studies have investigated the effectiveness of these gloves towards nanoparticle suspensions. Moreover, the data that are available are often contradictory. This study was designed to evaluate the effectiveness of protective gloves against nanoparticles in suspension. For this purpose, a new methodology was developed in order to take into account parameters encountered in the workplace such as mechanical deformations (MD) that simulate hand flexion and sweat. The effects of the precise experimental protocol on the concentrations of nanoparticles that were detected in the sampling suspension were assessed. Several samples of nitrile rubber gloves (73 $\mu \mathrm{m}$ thick), taken from different boxes, were brought into contact with gold nanoparticles $(5 \mathrm{~nm})$ in water. During their exposure to ENPs, the glove samples submitted systematic mechanical deformations and were placed in contact with a physiological solution simulating human sweat. Under these conditions, results obtained by inductively coupled plasma mass spectrometry (ICPMS) showed that the $5 \mathrm{~nm}$ gold nanoparticles passed through the protective gloves. This result was acquired, in spite of the observation of significant losses during the sampling phase that will be important for future experiments evaluating the effectiveness of these materials.
\end{abstract}

KEYWORDS: Nitrile rubber glove effectiveness, gold nanoparticle suspension, work conditions, human sweat, mechanical deformations. 



\section{INTRODUCTION}

The first evaluations of the effectiveness of disposable protective gloves against engineered nanoparticles (ENPs) began only recently. Indeed, upon confirmation of the toxicity of some ENPs (1-4) and the possibility that they could penetrate the skin (5-7), several Health \& Safety agencies have recommended the use of protective gloves even no thorough scientific validation of the gloves' effectiveness against nanoparticles had been made. As a result, a small number of research teams around the world began to take an interest in this problem.

Although the different particle compositions and phases of application (gas, liquid) may indeed lead to different mechanisms for penetrating protective materials, substantial differences observed todate in the literature can also be attributed to a lack of normalized experimental protocols for making quantitative determinations of gloves' effectiveness.

The evaluation of protective gloves against nanoparticles was first examined by Golanski et al. (8), who conducted permeation tests on as nitrile rubber, latex, neoprene and vinyl protective gloves. Airborne graphite nanoparticles of 30 to $80 \mathrm{~nm}$ in diameter were generated in a bench test without a significant air flow, in order to impose a diffusion regime. Diffusion coefficients were only measured for the $80 \mathrm{~nm}$ particles. Shortly thereafter, the same authors reported no permeation of graphite nanoparticles $(40 \mathrm{~nm})$ through the same type of glove, subjected to the same experimental conditions (9). One year later, Park et al. (10) studied the passage of airborne silver nanoparticles (range from 10 to $150 \mathrm{~nm}$ ) through nitrile rubber and latex gloves using a similar experimental approach. They concluded that no permeation of ENP occurred through the tested gloves. These above studies were conducted only with airborne nanoparticles and under low flow condition $(1 \pm 0.2 \mathrm{~L} / \mathrm{min})$, without 
dynamic mechanical deformations. More recently, Vinches et al. $(11,12)$ evaluated the effectiveness of nitrile rubber gloves with respect to the permeation of titanium dioxide and gold nanoparticles in water while applying cyclic 3D-deformations for a period of three hours. In that case, analysis by inductively coupled plasma mass spectrometry (ICPMS) analyses significant passage of the ENP, which led the authors to recommend regularly changing one's protective gloves when handling ENP.

This paper presents a rigorous experimental protocol that can be used in order to evaluate the effectiveness of disposable protective gloves against ENPs under conditions simulating occupational use.

\section{METHODS}

\section{Materials}

Disposable gloves (model Showa Best 3005PF Nitri-Care) with a thickness of $73.2 \pm 3.0 \mu \mathrm{m}$ were selected for this study. These are powder-free, $100 \%$ nitrile rubber protective gloves. To ensure statistical reproducibility, samples were taken from different batches and different boxes. Below, the gloves have been labelled as NBR-1a (batch 1, box a, manufactured in February 2015), NBR-1b (batch 1, box b, manufactured in February 2015), NBR-2 (batch 2, manufactured in September 2015) and NBR-3 (batch 3, manufactured in March 2014).

Gold nanoparticles (5 $\mathrm{nm}$ polyvinylpyrrolidone coated) suspended in Milli Q water were purchased from Nanocomposix (NanoXact grade, San Diego, CA). The gold concentration was 0.05 $\mathrm{mg} / \mathrm{mL}$ and commercial suspensions were used directly, without any dilution. The stability of the 
suspension is guaranteed by the supplier for 6 months and all suspensions are stored at $4^{\circ} \mathrm{C}$, away from light. Gold nanoparticles were used as a model ENP because they were easy to detect and they not enter in the chemical composition of the glove material. Furthermore their spherical shape allows a greatest degree of permeation through glove samples (13).

Although commercial solutions of artificial human sweat are available, all saline solutions used in this work were made in compliance with standard EN 1811 (14). The chemical composition of the solution was $0.5 \%(\mathrm{w} / \mathrm{w})$ sodium chloride, $0.1 \%(\mathrm{w} / \mathrm{w})$ lactic acid and $0.1 \%(\mathrm{w} / \mathrm{w})$ urea (14). The $\mathrm{pH}$ of human sweat ranges from 4.0 to 7.0 depending on the zone in the hand that is sampled, and of course the individual (15). In our case, the $\mathrm{pH}$ was adjusted to 6.0 using a $1 \%$ solution of sodium hydroxide. A fresh batch of physiological solution was prepared every 3 days and stored at $4^{\circ} \mathrm{C}$, protected from the light. The saline solution was removed from the refrigerator and maintained at ambient temperature $\left(22^{\circ} \mathrm{C}\right)$ for 30 minutes prior to the test.

\section{Experimental setup and experimental protocol}

A test setup was developed and is illustrated in Figure 1a. It includes an exposure chamber and a sampling chamber, which are separated by the glove sample and two seal rings (Figure 1b). The experimental setup is also equipped with a probe linked to an electronic system (actuator), which is used to apply the mechanical deformations to the sample. The system is computer-controlled and includes a $200 \mathrm{~N}$ load cell as well as a position detector. The entire system is enclosed within a glove box in order to ensure the operator's safety during the tests. All elements in contact with the ENPs (both chambers and the probe) are made of ultrahigh 
molecular weight polyethylene in order to limit absorption of the ENP by the contact surfaces. As seen in Figure 2, the glove sample is simultaneously in contact with two solutions: the ENP suspension $(10 \mathrm{~mL})$ is in contact with the external surface and the physiological solution $(90 \mathrm{~mL})$ is in contact with the internal surface.

To simulate hand and finger flexion, the probe was equipped with a $35 \mathrm{~mm}$ cone head with a spherical tip as described by Dolez et al. (16). The time profile of the MD consisted in $30 \mathrm{~mm}$ outof-plane deformations that were applied every 10 seconds over 3 hours with a set speed of $500 \mathrm{~mm} / \mathrm{min}$.

One main goal was to minimize any gold contamination in the sampling chamber. Contamination can come from a previous experiment or from a rupture of the glove sample. Since gold concentrations measured in the sampling solution could be relatively low $(\mu \mathrm{g} / \mathrm{L}$ or $\mathrm{ng} / \mathrm{L})$, appropriate care must be taken in cleaning the sampling chamber. Therefore, cleaning of the chambers was performed using four steps: 1) quick rinse under running water; 2) immersion in 2\% nitric acid and sonication (Fisher brand FB $11207-80 \mathrm{~Hz})$ for 20 minutes; 3) a second sonication in Milli Q water $(18.2 \mathrm{M} \Omega \cdot \mathrm{cm}$ and $25^{\circ} \mathrm{C}$, Organic Carbon $\left.<2 \mu \mathrm{g} \mathrm{C} \cdot \mathrm{L}^{-1}\right)$ for 10 minutes; 4) drying with an ultraclean air stream.

Before each test, $90 \mathrm{~mL}$ of a physiological solution was added to the sampling chamber and gently stirred in order to rinse the walls. This is the volume that is required in order to completely fill the sampling chamber and to maintain the glove sample in permanent contact with the solution. After 30 seconds, this solution was transferred to a bottle for ICPMS analysis and corresponds to the "blank sample". This procedure ensured that there was no contamination by ENP in the sampling chamber prior to the start of the test. An identical "test" solution ( $90 \mathrm{~mL}$ of physiological solution) was poured 
into the sampling chamber. The $10 \mathrm{~cm}$ diameter circle was sampled from the palm or the back section of a glove and positioned between the two chambers (Figure 2). To ensure an airtight seal between both solutions (physiological solution and gold nanoparticle suspension), two series of rings (neoprene and butyl rubber) were used to grip and seal the samples (Figure 1b). The sample and the seals were placed under the probe and $10 \mathrm{~mL}$ of the nanoparticle suspension was added to the exposure chamber in order to completely cover the glove sample (Figure 2).

When the test was completed, the sample, identified as "physiological solution" in Figure 2, was collected into a bottle made of a material that was compatible with the type of ENP being tested (see section 'Selecting compatible storage bottles') and the remaining nanoparticle suspension was recovered in waste bottles. The cell was then disassembled and thoroughly washed, according to the cleaning protocol described above and the sampling solution was analysed using ICPMS. In order to have statistically significant results, ten replicates were prepared for each batch of gloves.

\section{Characterization of the gold ENP and the nitrile protective gloves}

The distribution in size of the gold nanoparticles was verified using a statistical analysis of more than 100 particles imaged by TEM (Hitachi JEM-2100F). The hydrodynamic diameter and polydispersity index (PDI) were measured by dynamic light scattering (DLS - Mobius, Wyatt Technology). Nanoparticle electrophoretic mobilities (EPM) were measured by laser Doppler microelectrophoresis (Mobius, Wyatt Technology) and the zeta potential was determined by applying the Smoluchowski equation (17). For DLS and EPM measurements, independent triplicate measurements were performed. 
To investigate if gold nanoparticles were used in the manufacturing of the nitrile rubber gloves, a multi-element analysis was performed by ICPMS. Glove samples were immersed in physiological solution at $\mathrm{pH}-6$ for either 3 hours (approximate duration of the permeation tests). The swelling phenomenon is expected to partially degrade the material and thus release the reinforcing fillers used in its formulation (18). Results were presented as means \pm standard deviations $(\mathrm{n}=3)$.

\section{Selecting compatible storage bottles}

As indicated above, gold nanoparticles can be lost by absorptive losses during any step of the sampling protocol (from the sampling chamber to losses to tubing used during the ICPMS analysis). Thus, the chemical composition of the sampling bottles must be carefully chosen. In order to verify the compatibility between the sample bottles and the nanoparticles, a gold suspension at a nominal concentration of $10 \mu \mathrm{g} / \mathrm{L}$ (concentration expected based on previous work (12)) in physiological solution at $\mathrm{pH}-6$ was prepared and stored in containers of six different chemical compositions: glass, Teflon, polypropylene, polycarbonate, high density polyethylene and low density polyethylene. The day of the preparation, three volumes of $1 \mathrm{~mL}$ each were taken from each container after manual agitation. This procedure was repeated after 24, 48 and 72 hours. The samples were acidified with a 1\% ultrapure nitric acid (Fisherbrand) - 1\% ultrapure hydrochloric acid (Fisherbrand) prior the analysis of Au by ICPMS. All bottles were stored at $4{ }^{\circ} \mathrm{C}$ throughout the study.

\section{Evaluation of ENP losses}


Although the choice of the storage bottles and the choice of the sampling chamber material were optimized to reduce the loss of the gold nanoparticles by adsorption, numerous potential losses could occur during the sampling protocol. Three major sources of losses were identified: the sampling chamber, the storage bottles and during the ICPMS analysis (Figure 3).

In order to evaluate the losses that occurred during the sampling process, two suspensions of $5 \mathrm{~nm}$ gold nanoparticles $(10 \mu \mathrm{g} / \mathrm{L}$ and $100 \mu \mathrm{g} / \mathrm{L})$ were prepared in physiological solution at $\mathrm{pH}$ - 6. Using exactly the same experimental protocol as described before, three 'permeation' tests were carried out for each concentration, except that the physiological solution was replaced by a suspension containing a known concentration gold nanoparticles. In these tests, no nanoparticle suspension was placed in the upper exposure chamber. The recovered suspension was analyzed by ICPMS and a loss coefficient was calculated as follow:

$$
\text { Loss coefficient }=\frac{\text { gold concentration after the test }}{\text { initial gold concentration }} \times 100 \%
$$

\section{ICPMS analysis}

The samples were stored during a maximum period of 3 days before ICPMS analysis (PerkinElmer NexION 300X). As described in the section 'Selecting compatible storage bottles', $1 \mathrm{~mL}$ of the sampling solution was acidified with 1\%-nitric acid and 1\%-hydrochloric acid before analysis. All sampling solutions were stirred vigorously during 10 seconds. Quality control was performed by testing standards of the gold particles at known concentrations. A certified reference material was employed to verify the quality of the analysis. 


\section{RESULTS AND DISCUSSION}

\section{Initial characterization of gold ENP and nitrile protective gloves}

The size distribution of the gold nanoparticles was measured by TEM (Figure 4), giving physical particle diameters of $5.0 \pm 0.6 \mathrm{~nm}$. A hydrodynamic diameter $9.2 \pm 0.6 \mathrm{~nm}$ was obtained with a PDI of 0.016. The measured particle electrophoretic mobility was $-2.3 \pm 0.2 \mu \mathrm{m} \cdot \mathrm{cm} / \mathrm{s} \cdot \mathrm{V}$ giving a zeta potential of $-56.3 \pm 4.5 \mathrm{mV}$. These results confirmed the manufacturer's data.

In order to determine the amount of gold contained in the glove structure (no prior exposure to Au ENP), ICPMS analysis of the swelling solutions was performed. After 3 hours, the measured Au concentration was below the limit of detection (detection limits for $\mathrm{Au}$ of 1 to $3 \mathrm{ng} / \mathrm{L}$ ) and thus no interferences were expected for the measurements performed on solutions obtained from permeation tests.

\section{Selecting compatible storage bottles}

The assessment of the stability of the gold concentrations over time illustrates the importance of the choice of the storage bottles used during sampling. Gold concentrations detected in the sampling solutions after permeation tests were relatively low and in most cases under $10 \mu \mathrm{g} / \mathrm{L}$ (see section 'Gold nanoparticle permeation: ICPMS results').

Loss coefficients for the gold ENP due to absorption to the walls of the storage bottles were significant, even 24 hours after the preparation of the suspensions, except in the case of the glass bottles (where the loss coefficient remained at 0\%) (Table I). Furthermore, 24 hour loss coefficients reached 
$50 \%$ for bottles made from high density polyethylene (HDPE). After a contact time of 72 hours, the coefficient remained at $0 \%$ for glass bottles but increased to $70 \%$ for HDPE. For these reasons, in the remainder of the study, all prepared suspensions and sampling solutions were stored in glass bottles.

\section{Further losses of Au ENP}

The judicious choice of sample storage bottles partially (or totally in the case of glass bottles) reduced the amount of gold lost by adsorption to the sampling bottles. However, at other points in the sampling protocol (Figure 3), the loss coefficient reached $51.0 \pm 0.1 \%$ for low gold concentrations $(\sim 10 \mu \mathrm{g} / \mathrm{L})$ and $41.0 \pm 0.1 \%$ for high gold concentrations $(-100 \mu \mathrm{g} / \mathrm{L})$. As described in the previous section, the gold concentrations detected in the sampling solutions after permeation tests were low and, in most cases, below $10 \mu \mathrm{g} / \mathrm{L}$. Thus, the higher loss coefficient was likely more realistic for the permeation tests.

\section{Gold nanoparticle permeation: ICPMS results}

First, to ensure that any gold measured in the sampling solutions was present as a result of the passage of nanoparticles, blank tests (in the absence of the Au ENP) were performed by applying the same experimental protocol as the one used for the permeation tests. In all of these "blank" tests, gold concentrations were below the limit of detection (LOD $=1$ to $3 \mathrm{ng} / \mathrm{L}$ ) of the ICPMS. Mean concentrations measured for four different batches and boxes of gloves for tests conducted with gold nanoparticle suspensions and the different glove samples are presented in Table II. For each sample, 10 tests were performed, for a total of 40 tests. 
The results confirm the passage of gold nanoparticles through this model of nitrile rubber gloves and indicate its low effectiveness against these nanoparticles. Moreover, significant differences in extent of permeation were observed depending on precise glove sample studied. For NBR-1a and NBR-1b (same batch but two different boxes), the maximum concentration differed by approximately $230 \%$ and for different boxes (NBR-1a and NBR-2), a 13 fold difference in the maximum measured gold concentrations was observed. The best protection against gold nanoparticles was observed with the oldest batch of gloves (NBR-3). Figure 5 shows the distribution in Au concentrations for all of the 40 tests. Although a majority of concentrations measured were less than $0.500 \mu \mathrm{g} / \mathrm{L}$, it was impossible to determine a statistical profile of this distribution. The disparities in the concentration measurements, large standard deviations and inability to determine a statistical distribution of gold concentrations strongly suggest some variability in glove manufacturing process, at least for this model.

\section{CONCLUSION}

This study has established a new experimental methodology to evaluate the effectiveness of protective gloves against nanoparticles. This work identifies several important steps of the experimental protocol:

1- Determine the most suitable storage bottles for the sampling solution.

2- Evaluate the loss coefficient of the sampling protocol.

3- Clean the different parts of the test setup to minimise contamination.

4- Measure the permeation of ENPs through disposable protective gloves. 
Moreover, this methodology takes into account certain conditions encountered in the workplace, particularly mechanical constraints and the sweat on the performance of the protective gloves during their use. Indeed, the test setup was developed to simulate the movements of the hand flexing and also to partially reproduce the microclimate within the glove (sweat).

For the same glove model, the results were variable depending on the batch and on the box from which the gloves were taken. This could be due to variability in the manufacturing process for these disposable nitrile gloves. Nonetheless, in all cases, significant concentrations of gold nanoparticles were observed in the sampling solution. The permeation of ENPs was likely due to a loss of integrity of the elastomer structure caused by the mechanical deformations and by the swelling of the sample in contact, at the same time, with both the gold nanoparticles and the physiological solution (19).

Although this methodology includes several stages, it represents a sound basis towards a validated protocol to evaluate the effectiveness of disposable protective gloves against ENP permeation. 


\section{ACKNOWLEDGEMENTS}

This research was supported by the Institut Robert-Sauvé de recherche en santé et sécurité du travail

(IRSST) and Prima Québec. The authors acknowledge the technical contributions of Aoued Belhadj and Nidal Boutrigue (École de Technologie Supérieure) and Laura Lemarchand and Madjid Hadioui (Université de Montréal). 


\section{REFERENCES}

1. Sharifi, S., et al.: Toxicity of nanomaterials. Chemical Society Reviews. 41(6):2323-2343 (2012).

2. Singh, S. and H.S. Nalwa: Nanotechnology and health safety - Toxicity and risk assessments of nanostructured materials on human health. Journal of Nanoscience and Nanotechnology. 7(9):3048-3070 (2007).

3. Srivastava, V., D. Gusain, and Y.C. Sharma: A critical review on the toxicity of some widely used engineered nanoparticles. Industrial \& Engineering Chemistry Research. (2015).

4. Alkilany, A. and C. Murphy: Toxicity and cellular uptake of gold nanoparticles: what we have learned so far? Journal of Nanoparticle Research. 12(7):2313-2333 (2010).

5. Labouta, H.I., et al.: Mechanism and determinants of nanoparticle permeation through human skin. Nanoscale. 3(12):4989-4999 (2011).

6. Hirai, T., et al.: Potential Hazards of Skin Exposure to Nanoparticles. In Biological Effects of Fibrous and Particulate Substances, T. Otsuki, Y. Yoshioka, and A. Holian (ed.). Springer Japan: Tokyo, 2016. pp. 123-135.

7. Larese Filon, F., et al.: Nanoparticles skin absorption: New aspects for a safety profile evaluation. Regulatory Toxicology and Pharmacology. 72(2):310-322 (2015).

8. Golanski, L., A. Guiot, and F. Tardif: Are conventional protective devices such as fibrous filter media, respirator cartridges, protective clothing and gloves also efficient for nanoaerosols? Available at: http://www.nanosafe.org/home/liblocal/docs/Dissemination\%20report/DR1 s.pdf (accessed August 30, 2016)

9. Golanski, L., et al.: Experimental evaluation of personal protection devices against graphite nanoaerosols: fibrous filter media, masks, protective clothing, and gloves. Human and Experimental Toxicology. 28(6-7):353-359 (2009).

10. Park, J., et al.: Efficiency of protective dermal equipment against silver nanoparticles with water aerosol. Journal of Nanoparticle Research. 13(7):3043-3049 (2011).

11. Vinches, L., et al.: Experimental evaluation of the permeation of $\mathrm{TiO}_{2}$ nanoparticles through protective clothing and gloves under conditions simulating occupational use. Nanoscience Methods. 1-15 (2013).

12. Vinches, L., et al.: Effectiveness of Protective Gloves against Engineered Nanoparticles: Difficulties in Evaluation. International Journal of Theoretical and Applied Nanotechnology. 4:9-16 (2016).

13. Masaro, L. and X.X. Zhu.: Physical models of diffusion for polymer solutions, gels and solids. Progress in Polymer Science. 24(5):731-775 (1999).

14. E.C.F.: Reference test method for release of nickel from product intended to come in direct and prolonged contact with the skin: BS EN 1811:1998+A1:2008. 1998: Brussels CEN.

15. Lambers, H., et al.: Natural skin surface $\mathrm{pH}$ is on average below 5, which is beneficial for its resident flora. International Journal of Cosmetic Science. 28(5):359-370 (2006).

16. Dolez, P., et al.: Development of a test method for protective gloves against nanoparticles in conditions simulating occupational use. Journal of Physics: Conference Series. 304(1):012066 (2011).

17. Hunter, R., R. Ottewill, and R. Rowell: Zeta Potential in Colloid Science - Principles and Applications. New-York: Academic Press, 1981.

18. Nohile, C., P.I. Dolez, and T. Vu-Khanh: Parameters controlling the swelling of butyl rubber by solvents. Journal of Applied Polymer Science. 110(6):3926-33 (2008).

19. Vinches, L., et al.: Physical phenomena facilitating the permeation of solutions of $\mathrm{TiO}_{2}$ nanoparticles through protective gloves. International Journal of Theoretical and Applied Nanotechnology. 1(2):1-16 (2013). 


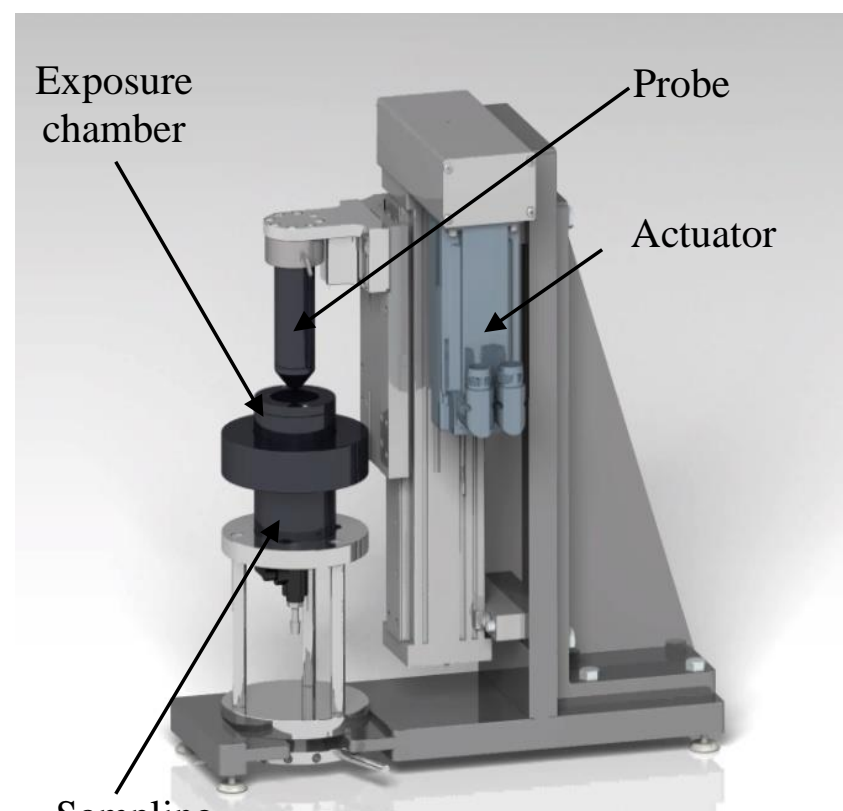

Sampling chamber

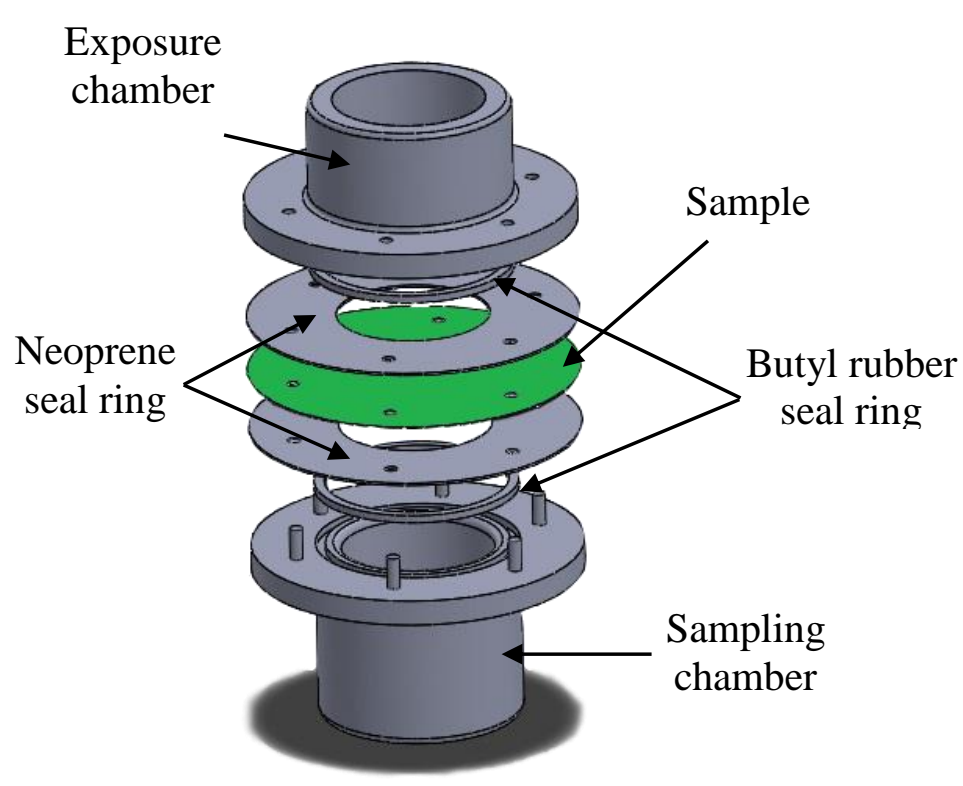

b)

FIGURE 1 a) Isometric view of the test setup and b) Exploded view of the sample positioned between the two chambers 


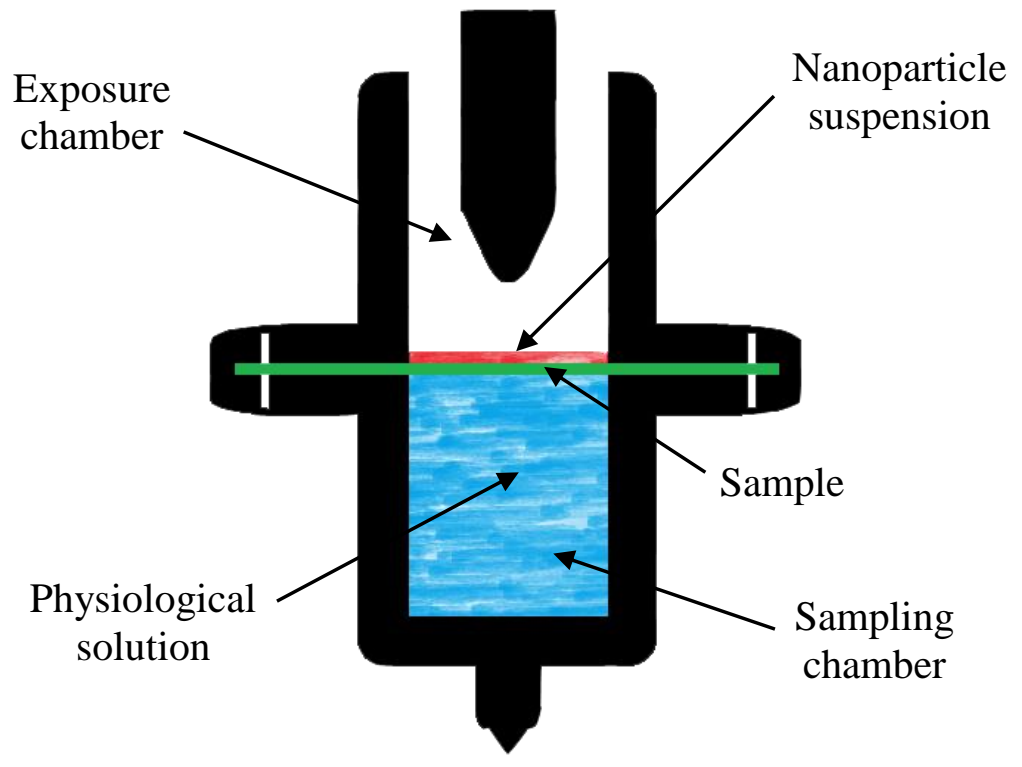

FIGURE 2 Schematic of both chambers before starting a permeation test 


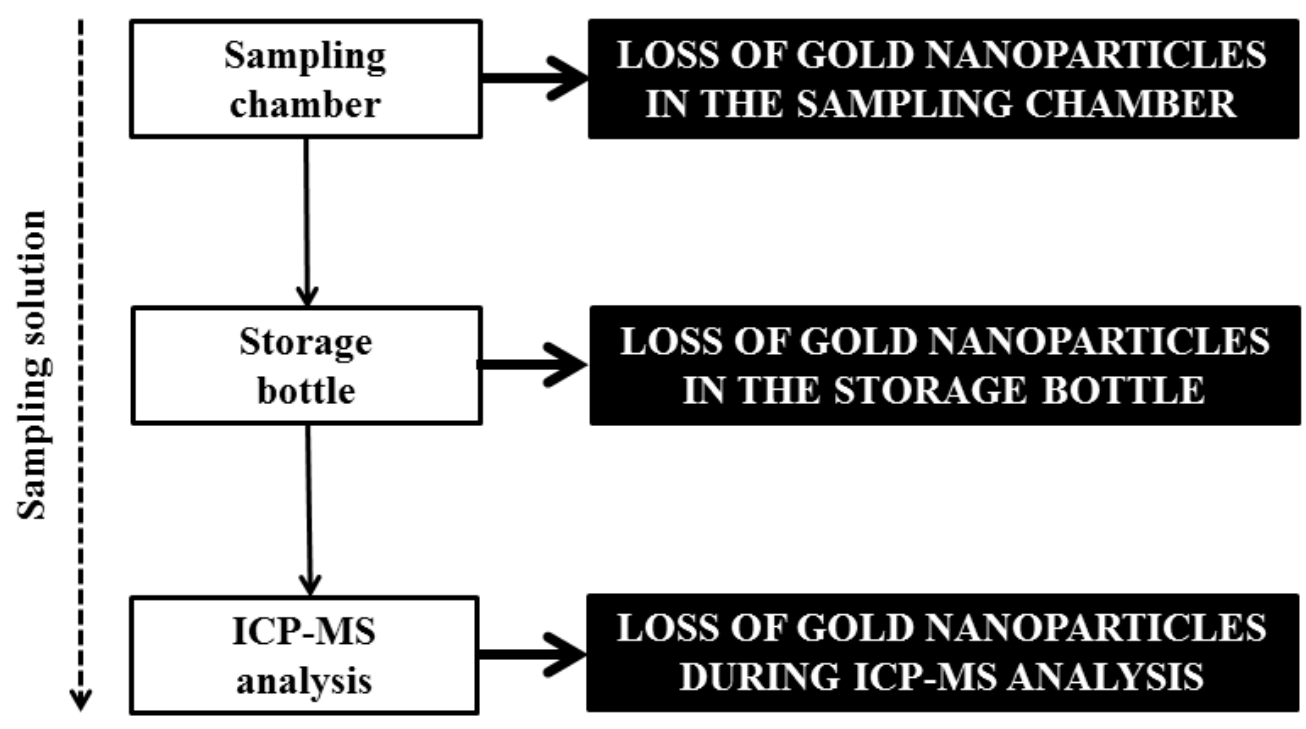

FIGURE 3 Sources of losses during the sampling protocol 


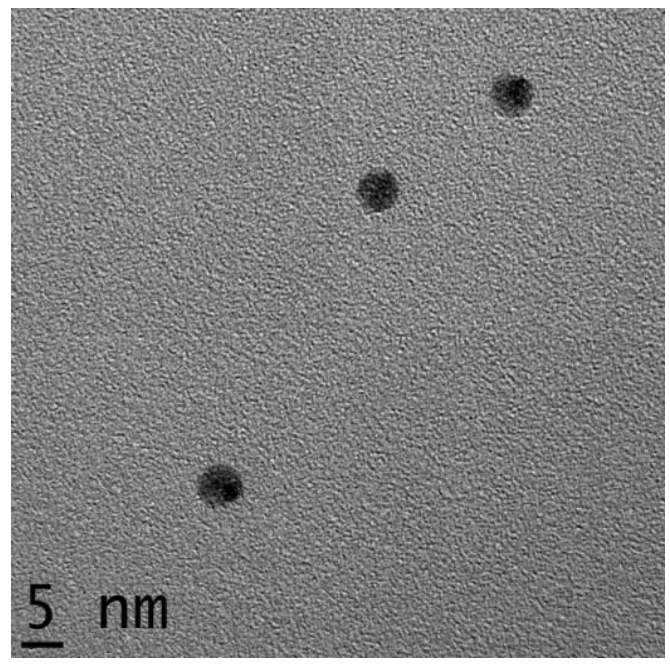

FIGURE 4 TEM image of the gold nanoparticle suspension 


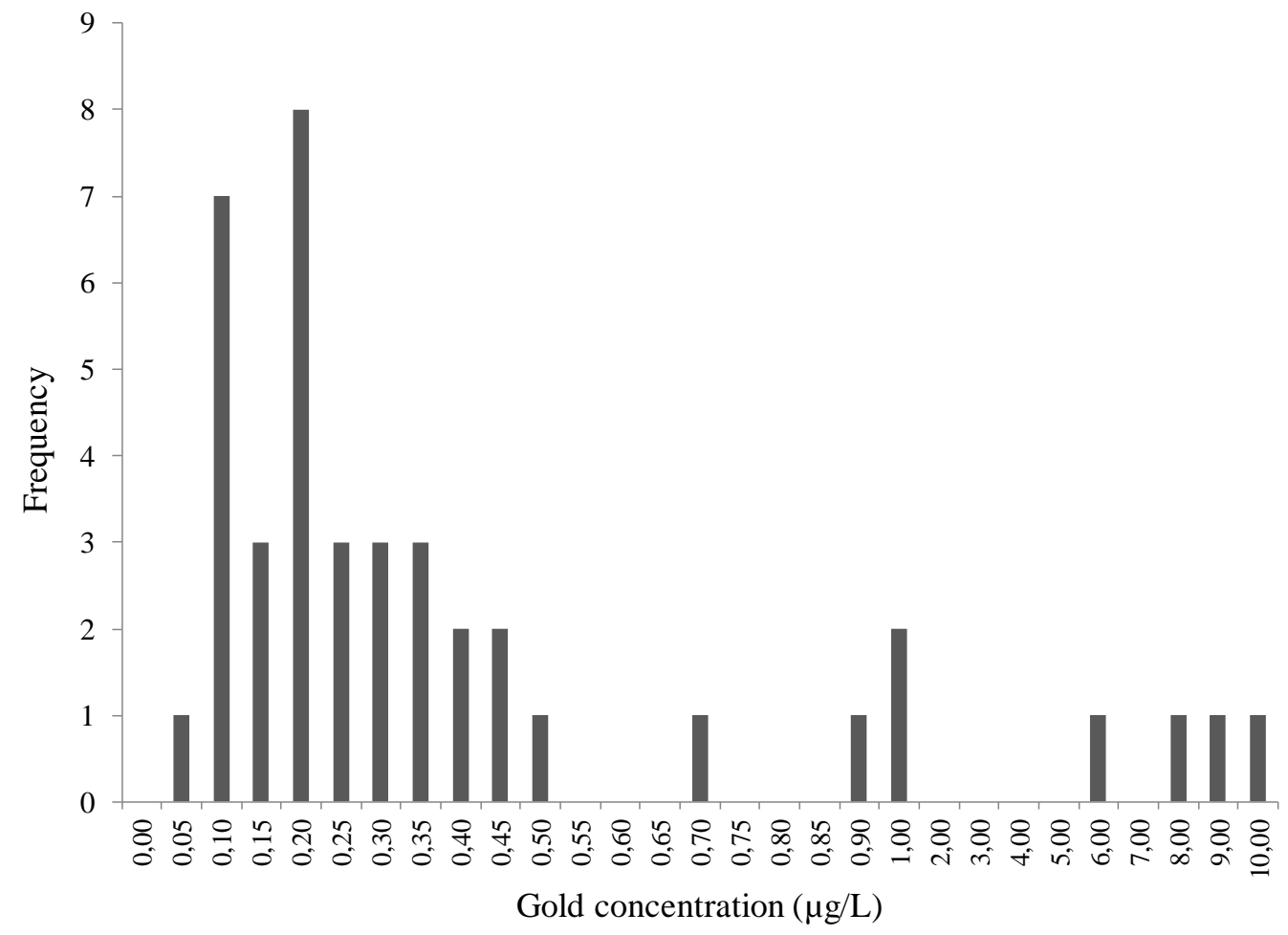

FIGURE 5 Distribution in gold concentration for all the tests performed 
TABLE I Loss coefficient (\%) of $\sim 10 \mu \mathrm{g} / \mathrm{L}$ gold suspension after 24 and 72 hours in different storage bottles

\begin{tabular}{ccc} 
& After 24 hours & After 72 hours \\
Glass & $\mathbf{0}$ & $\mathbf{0}$ \\
\hline Polycarbonate & 25 & 35 \\
\hline Polypropylene & 30 & 50 \\
\hline Teflon & 35 & 50 \\
\hline Low Density Polyethylene & 40 & 60 \\
\hline High Density Polyethylene & 50 & 70
\end{tabular}


TABLE II Gold concentrations $(\mu \mathrm{g} / \mathrm{L})$ measured in the sampling solution after the permeation test with nitrile rubber gloves from different boxes and batches.

\begin{tabular}{cc}
$\mathrm{nAu}-5$ & $\begin{array}{c}\mathrm{M} \pm \mathrm{SD} \\
(\mathrm{n}=10)\end{array}$ \\
\hline NBR-1a & $0.446 \pm 0.162$ \\
\hline NBR-1b & $0.530 \pm 0.524$ \\
\hline NBR-2 & $1.662 \pm 2.994$ \\
\hline NBR-3 & $0.273 \pm 0.132$
\end{tabular}

